\title{
Una reflexión sobre las movilidades e interacciones sociales en los lugares de transporte en tiempos de Covid-19: El caso del "Metropolitano", Lima - Perú
}

\section{A reflection on mobility and social interactions in places of transport in times of Covid-19: The case of the "Metropolitano", Lima - Perú}

\section{Uma reflexão sobre mobilidade e interações sociais em locais de transporte em tempos de Covid-19: O caso do "Metropolitano"}

\author{
Aroldo Kevin Chavez Leonardo \\ Universidad Nacional Federico Villarreal \\ kevin8aroldo@gmail.com
}

\begin{abstract}
RESUMEN
La actual pandemia por la Covid-19 ha repercutido en la manera de interactuar en diversos espacios de nuestra vida social, uno de estos espacios son las grandes estaciones de transporte de las muchas ciudades del mundo. En ese sentido, para el desarrollo de este texto, un sistema de autobuses de tránsito rápido que opera en la ciudad de Lima, el "Metropolitano", nos será de caso empírico para reflexionar sobre dicha repercusión. De esta manera, para entender dicha repercusión, en primer lugar, se centrará en las movilidades e interacciones sociales de los usuarios del Metropolitano hasta antes de la pandemia. Para así posteriormente, analizar de qué manera el presente contexto ha repercutido en la forma de interactuar en este sistema de transporte. En suma, para generar un mayor sustento a este texto, se emplearán y analizarán algunas respuestas que se han recopilado a través de un formulario virtual sobre la experiencia de viajar en el Metropolitano en esta pandemia. Así mismo, se describirán y analizarán algunos escenarios etnográficos que se han podido registrar, mediante la observación participante, en las estaciones del Metropolitano.
\end{abstract}

\begin{abstract}
The current Covid-19 pandemic has had an impact on the way we interact in various spaces of our social life, one of these spaces is the large transport stations in many cities in the world. In this sense, for the development of this text, a rapid transit bus system that operates in the city of Lima, the "Metropolitan", will be an empirical case to reflect on this impact. In this way, to understand said repercussion, in the first place, it will focus on the mobility and social interactions of the users of the Metropolitano until before the pandemic. To do so later, analyze how the present context has impacted on the way of interacting in this transport system. In short, to generate greater support for this text, some responses that have been compiled through a virtual form on the experience of traveling in the Metropolitan in this pandemic will be used and analyzed. Likewise, some ethnographic scenarios that have been recorded, through participant observation, in the Metropolitano stations will be described and analyzed.
\end{abstract}

Recibido:27/08/2020 - Aceptado: 08/09/2020 - Publicado:22/02/2021

Citar como:

Chavez, A. (2020). Una reflexión sobre las movilidades e interacciones sociales en los lugares de transporte en tiempos de Covid-19: El caso del "Metropolitano", Lima - Perú. Espiral, revista de geografías y ciencias sociales, 2(4), 053 - 068. http://dx.doi.org/10.15381/espiral.v2i4.19533 


\section{RESUMO}

A atual pandemia de Covid-19 teve um impacto na forma como interagimos em vários espaços de nossa vida social, um desses espaços são as grandes estações de transporte em muitas cidades do mundo. Nesse sentido, para o desenvolvimento deste texto, um sistema de ônibus de trânsito rápido que opera na cidade de Lima, o "Metropolitano", será um caso empírico para refletir sobre esse impacto. Dessa forma, para compreender tal repercussão, em primeiro lugar, enfocará as mobilidades e interações sociais dos usuários do Metropolitano até antes da pandemia. Para fazer isso posteriormente, analise como o contexto atual impactou na forma de interagir neste sistema de transporte. Em suma, para gerar maior sustentação a este texto, serão utilizadas e analisadas algumas respostas que vêm sendo compiladas por meio de um formulário virtual sobre a experiência de viajar no Metropolitano nesta pandemia. Da mesma forma, serão descritos e analisados alguns cenários etnográficos que foram registrados, por meio da observação participante, nas estações do Metropolitano.

PALABRAS CLAVE: Interacciones sociales; Covid-19; Metropolitano; ciudad; transporte; movilidades.

KEYWORDS: Social Interations; Covid-19; Metropolitano; city; transport; mobilities.

PALAVRAS-CHAVE: Interação social; Covid-19; Metropolitano; cidade; transporte; mobilidade.

\section{Introducción}

En el espacio urbano de la ciudad se desenvuelven una serie de dinámicas que permiten el desenvolvimiento de otras actividades sociales; por ejemplo, las grandes estaciones de transporte público y masivo se representan como un espacio de puente o de paso hacia otras actividades de mayor relevancia social para las personas, como: el centro de trabajo, de estudios o de otra actividad social. Esta cuestión mecánica y cotidiana de transportarse de un lugar a otro o de una estación a otra, por lo general, determina que el usuario o el pasajero observe a esto como la única función del transporte. No obstante, para un sector de las ciencias sociales, dichos espacios son relevantes porque permiten identificar en las movilidades e interacciones sociales el significado y sentido social que estos lugares representan en la dinámica urbana de la ciudad.

Perteneciendo a este sector de las ciencias sociales se encuentran: interaccionistas simbólicos como Erving Goffman $(1970,2001)$ y su observación hacia los seres humanos como actores que desenvuelven diversos rostros, mascarás y comportamientos en el transcurso de las relaciones sociales con otras personas, así como en diversos escenarios cotidianos. En otras palabras, los estudios de Goffman se centran en lo que él denomina como la "sociología de las ocasiones".

Los estudios de Howard Becker y su interés por lo que él denomina "sociología de la desviación" (2014). Es decir, grupos minoritarios de la ciudad que desenvuelven actividades no comunes o particulares, como bien se puede observar en sus estudios sobre los consumidores de marihuana y los músicos de jazz (2006), así como también grupos diferentes o "raros" que conforman parte del estudio que el autor denomina como "outsiders" (2014). El interés de este autor por la importancia de estos tipos de estudios se puede observar en un libro-manual que H. Becker (2009) elabora para aquellos estudiantes o investigadores que tienen el interés de realizar investigaciones de este tipo.

Por su lado, Edward T. Hall (2003) analiza la manera en que los individuos ejercen sus espacios personales en medio de la cotidianidad de sus actividades sociales. Para Hall, este tipo de estudio es lo que él denomina como la "proxémica". Así mismo, este autor profundiza entre las diferentes normales culturales que los grupos, sociedades o países ejercen sobre el espacio de la ciudad, así como de las prolongaciones del organismo de las personas -mediante la tecnología- en sus interacciones que él entendía como aquella dimensión cultural (oculta) de los grupos sociales. Aquella 
dimensión oculta me hace recordar y relacionar con ese "algo más" que para Daniel Hiernaux (2012) simboliza al "verdadero espíritu de la ciudad" o la "personalidad de la ciudad".

Desde una mirada biopolítica o del biopoder, Michael Foucault (2010) aborda sobre el cuerpo utópico y los lugares heterotópicos de la ciudad. Entendiendo al cuerpo utópico como la capacidad simbólica que tiene para adoptar nuevas formas de presentarse, sean visibles o no visibles, en un lugar y de experimentar sensaciones particulares en distintos lugares por donde el cuerpo transité. En estos distintos lugares del espacio de la ciudad, para el autor, se originan las heterotopías: lugares yuxtapuestos, desviados o diferentes que Foucault afirma que toda cultura ha debido tener. Estos lugares heterotópicos ayudaron al autor a analizar la territorialidad del espacio de la ciudad, así como de las relaciones de poder que pueden producirse en estos "espacios diferentes".

Otro autor que fue muy relevante para comprender al espacio de la ciudad ha sido Henri Lefebvre (2013), su importante análisis nos invita a observar a la ciudad como un espacio ideológico y de disputas de clases. Así como para reconocer, críticamente, a aquellos que diseñan y estructuran el espacio y las normas de la ciudad desde lo cotidiano hasta lo más complejo de las instituciones del estado como de lo privado (burguesía). Aun así, la complejidad de Lefebvre es mucho más ya que también ahonda en el plano filosófico y metafísico de la ciudad. En la misma línea de este tipo de análisis, se encuentra Manuel Delgado, siendo un autor muy importante debido a sus estudios sobre el espacio como ideología (2011) en donde analiza al espacio, inspirado por Lefebvre, de la ciudad como extensión material e ideológica de los que diseñan y organizan el espacio de la ciudad. En donde el Estado y su legitimación simbólica, según el autor, son el determinante para enmascarar y soslayar ante la población el verdadero propósito y rostro de ese sector coludido política y socialmente. Pero que aún con esa legitimación, el espacio público es un escenario de constante disputas y contradicciones, simbolizando y desenmascarando así la existencia de las asimetrías sociales. Así mismo, Delgado analiza sobre la persona, el sujeto social, el usuario o el autor que interactúa cotidianamente por los lugares urbanos de la ciudad, aquel "animal público" (1999) que ha sido de interés por la sociología de las interacciones o microsociología, es analizado por Delgado de una manera tan detallada y minuciosa que nos ha permitido introducirnos en una especie de línea de vida sobre el estudio de las interacciones sociales y de lo que pasa u ocurre en lo cotidiano de la ciudad.

En otra línea, el antropólogo francés, Marc Augé, ha realizado diversos análisis teóricos $(1998,2009)$ de este tipo de espacio de tránsito rápido y de paso, como fue con el Metro de Paris. Para este autor, estos tipos de escenarios en la actualidad -o lo que él denomina como "sobremodernidad"- no presentan algún tipo de relevancia social para considerarlos lugares, ya que no cuentan con las tres características (histórico, relacional, identitario) que para Augé hacen a un lugar. Es así que, para el autor, estos lugares son denominados "no-lugares" (1998), es decir, espacios no antropológicos.

Por otro lado, fuera de lo puramente teórico (eso no quiere decir que los autores anteriores no se hayan basado en estudios empíricos), centrándonos en la línea de nuestro caso empírico en reflexión, hasta el momento se ha recopilado algunos textos de estudios de casos de lugares de transporte masivo y rápido, como los trenes y autobuses de líneas extensas. Estos tipos de transporte suelen ocupar grandes extensiones de espacios de la ciudad ya que conectan a muchas calles, avenidas y distritos donde parte de la cotidianidad es transitar por estas, ya que son un puente para dirigirse al trabajo, a la universidad, a un evento social, etc. Estas etnografías también realzan la importancia de las interacciones sociales en estos espacios de tránsito rápido por ser lugares simbólicos que cuentan con sus propias dinámicas internas. Dichos casos pueden catalogarse dentro de la "antropología de la movilidad" que Delgado (1999) acude para hacer referencia a la ciencia social de la antropología urbana. 
Por ejemplo, sobre el Metro de la Ciudad de México se ha podido encontrar estudios sobre las interacciones sociales que ocurren en los vagones del metro, así como de prácticas sociales que han marcado un significado en el espacio del metro (Aguilar, 2013). Estas prácticas, para los autores, reconfiguran el significado del metro como únicamente de transporte; y, por el contrario, se descubre esa otra esencia social del metro producto de estas interacciones sociales. Por ejemplo prácticas como las "relaciones homoeróticas" (Hernández, 2017) y el "metreo" (Nava, 2019) que grupos de hombres homosexuales lleva a cabo en el último vagón del metro de México como besos, roces, penetración, miradas, toqueteos, etc., son sumamente relevantes porque son situaciones que dan sustento empírico a esa reconfiguración del significado del metro: como un lugar que produce en su interioridad una propia dinámica social.

En nuestro caso local, en Perú, existe un estudio de la Línea 1 del Metro de Lima (Vivanco, 2019) en donde el autor describe la historia y dinámica social del metro, y analiza a partir de estos la idea de cómo poder construir una mejor ciudadanía. Por otro lado, hay un estudio donde se aborda a un sector del transporte público, las combis medio formal e informal, de la ciudad de Lima en la madrugada (Toledo, 2014). Aunque su escenario de estudio fue relativamente diferente, consideramos importante en la medida que podemos rescatar ciertas interacciones sociales que no suelen ocurrir en el transporte diurno y que por el contrario manifiestan su propia dinámica social por las altas horas de la noche. Estos casos citados nos estarían demostrando que "el tránsito representa más que la acción física [y mecánica] de trasladarse entre dos puntos del plano terrestre" (Grimaldo, 2020, p.2).

De esta manera se espera que este breve estado de cuestión haya sido de ayuda al lector o lectora para introducirse y, sobre todo, despertar el interés sobre estos tipos de espacios de la ciudad, que, aunque no fueron realizados y analizados en un contexto de pandemia, no deja de ser notable al momento de pensar socialmente la ciudad.

\section{Metodología}

Para el desarrollo y sustento del presente texto, se ha realizado una observación participante ${ }^{1}$ por las diversas estaciones del Metropolitano, pero principalmente en el Terminal de transferencia Naranjal. Este trabajo de campo ${ }^{2}$ ha permitido capturar y anotar los diversos escenarios de movilidad e interacciones sociales que los usuarios del Metropolitano desenvuelven como parte de la -en el sentido colectivo- y su -en el sentido individual- cotidianidad por este servicio de transporte. Así mismo, se ha prestado atención a las emociones y sentimientos corporalizados (Lindón, 2009) en los usuarios en aquellos escenarios y momentos de mayor agudización simbólica, es decir, en las horas punta

Por otro lado, los comentarios en las publicaciones de la página de Facebook del Metropolitano por parte de los cientos de usuarios, es idóneo porque consideramos que el plano virtual es una oportunidad para rescatar la opinión que tienen los usuarios hacia este tipo de transporte que difícilmente lo expresarían en palabras un lunes por la mañana en plena hora punta, donde la preocupación mayor está en llegar al trabajo, estudio, etc. De hecho, gracias al plano virtual, se ha realizado también un formulario virtual ${ }^{3}$ en donde una de las once preguntas estuvo relacionado a la experiencia de viajar y transitar en el Metropolitano en el presente contexto de pandemia. De esta manera, las expresiones escritas recogidas mediante este

\footnotetext{
1 Entendemos por observación participante como Tim Ingold (2017) lo define: "Observar significa ver lo que está sucediendo alrededor, y por supuesto, también escuchar y sentir. Participar significa hacerlo dentro del flujo de actividades en las cuales uno lleva la vida, al lado de y junto de las personas y cosas que capturan la atención" (pág. 148).

2 Nos sostenemos en la siguiente cita de Eduardo Restrepo (2018): "El trabajo de campo se refiere a esa fase del proceso investigativo dedicado al levantamiento de la información requerida para responder a un problema de investigación. El trabajo de campo es el momento en el cual el etnógrafo realiza el grueso de la labor empírica” (pág. 51).

3 Aunque dicho formulario virtual estuvo pensado para una futura investigación y sin miras a esta pandemia; no obstante, no pudimos ser ajeno a realizar una pregunta que girará en torno al actual contexto.
} 
formulario, que se transcribirán en el desarrollo del texto, servirán para analizar los sentires de los usuarios del Metropolitano en el actual contexto.

En suma, para este texto se ha contado también con el análisis de los imaginarios urbanos e interacciones intersubjetivas (Hiernaux, 2012) para entender al Metropolitano como un micro-lugar donde se prolongan los problemas urbanos ${ }^{4}$ de la ciudad de Lima. Por otra parte, está pendiente el realizar entrevistas de manera presencial a actores sociales que son usuarios de este servicio de transporte, y que además poseen ideas críticas al tipo de servicio del Metropolitano desde una mirada social, ambiental y política.

\section{Breve descripción de la morfología física del Metropolitano}

Para que el lector o la lectora pueda recrear imaginariamente el espacio del cuál se compone el Metropolitano, se ha considerado necesario realizar una breve descripción de su morfología física. Es decir, de la infraestructura material que es el escenario para las movilidades e interacciones sociales que se producen en su interior. Ya Marc Augé recomendaba la importancia de iniciar con la descripción de estos tipos de lugares para poder comprender el funcionamiento de un transporte público de alguna ciudad.

[...] una gran estación, de una estación con empalmes y conexiones, debería comenzar por una descripción metódica de los lugares... De manera... que señalara la articulación de los corredores de dos manos o de mano única que permiten llegar a cada uno de ellos y pasar de uno a otro, que distinguiera y situara exactamente la entrada principal y las demás entradas, las ventanillas donde se venden los billetes y las tarjetas de abono, que apreciara la dificultad de acceso a los diferentes puntos en que se toman los trenes, la longitud de los corredores y de las escaleras... [es decir] la morfología física (Augé, 2009, p.45).

De esta manera, el transporte público, urbano y masivo del Metropolitano que opera en la ciudad de Lima desde el 2010, es un sistema de rutas troncales-alimentadores que se conforman por dos terminales de transferencias (Terminal Naranjal y Matellini), 35 estaciones y una estación central subterránea. Este sistema de transporte cuenta con una vía exclusiva que conecta a 18 distritos (ver figura 1$)^{5}$ de la ciudad de Lima. Una vía que desde luego es público si se entiende que es solo para los buses del Metropolitano o para las ambulancias, bomberos y policías cuando están en situación de emergencia; más no para los otros tipos de transporte tradicional, medio formal e informal ${ }^{6}$.

Las 35 estaciones comparten, por lo general, la misma infraestructura (ver figura 2) salvo en algunas diferencias por las dimensiones ya que en algunas estaciones se recibe a una mayor cantidad de usuarios. Por ejemplo, en el Terminal Naranjal (Lima Norte), en el Terminal Matellini (Lima Sur) y la Estación Central (Lima Centro) cuentan con más rutas, servicios higiénicos y es mucho más amplio (ver figura 3) ya que son los puntos de llegada y salida tanto de buses como de alimentadores.

La interioridad de los terminales y estaciones del Metropolitano cobran sentido y vida social desde tempranas horas de la mañana hasta altas horas de la noche. Siendo así, que algunas estaciones del Metropolitano poseen una mayor carga simbólica y social debido a su mayor interacción y aglomeración de sucesos por la cantidad de usuarios que se movilizan. Así como en otras estaciones la capacidad de aglomeración rebasa, en el Terminal Naranjal también ocurre ya que es un punto de mayor aglomeración 4 Como marchas, protestas y plantones que tuvieron como escenario al mismo espacio del Metropolitano hasta antes de la pandemia.

5 En la figura 1 solo se podrá presenciar a 12 distritos ya que están incluidos en la vía exclusiva, más no las 8 restantes debido que estos distritos empiezan al margen de los dos terminales de transferencia como también de la Estación Central. 6 Esta es la razón por la cual en horarios punta el Metropolitano es una “alternativa” para transportarse rápidamente por su vía exclusiva, mientras que los otros tipos de transporte tienen que lidiar con el tráfico, embotellamientos o aglomeración en diversos puntos de la ciudad, como sucede en la Av. Túpac Amarú de Lima Norte. Esta avenida que colinda con la vía exclusiva del Metropolitano desde el Terminal Naranjal del distrito de Independencia hasta la estación UNI del distrito de San Martin de Porres, es un caso empírico para contrastar en el mismo espacio a dos tipos de transporte urbano de la ciudad de Lima. 
de usuarios, esto es clave para aproximarnos a entender todo ese flujo de movilidades e interacciones sociales que da sentido al Metropolitano.

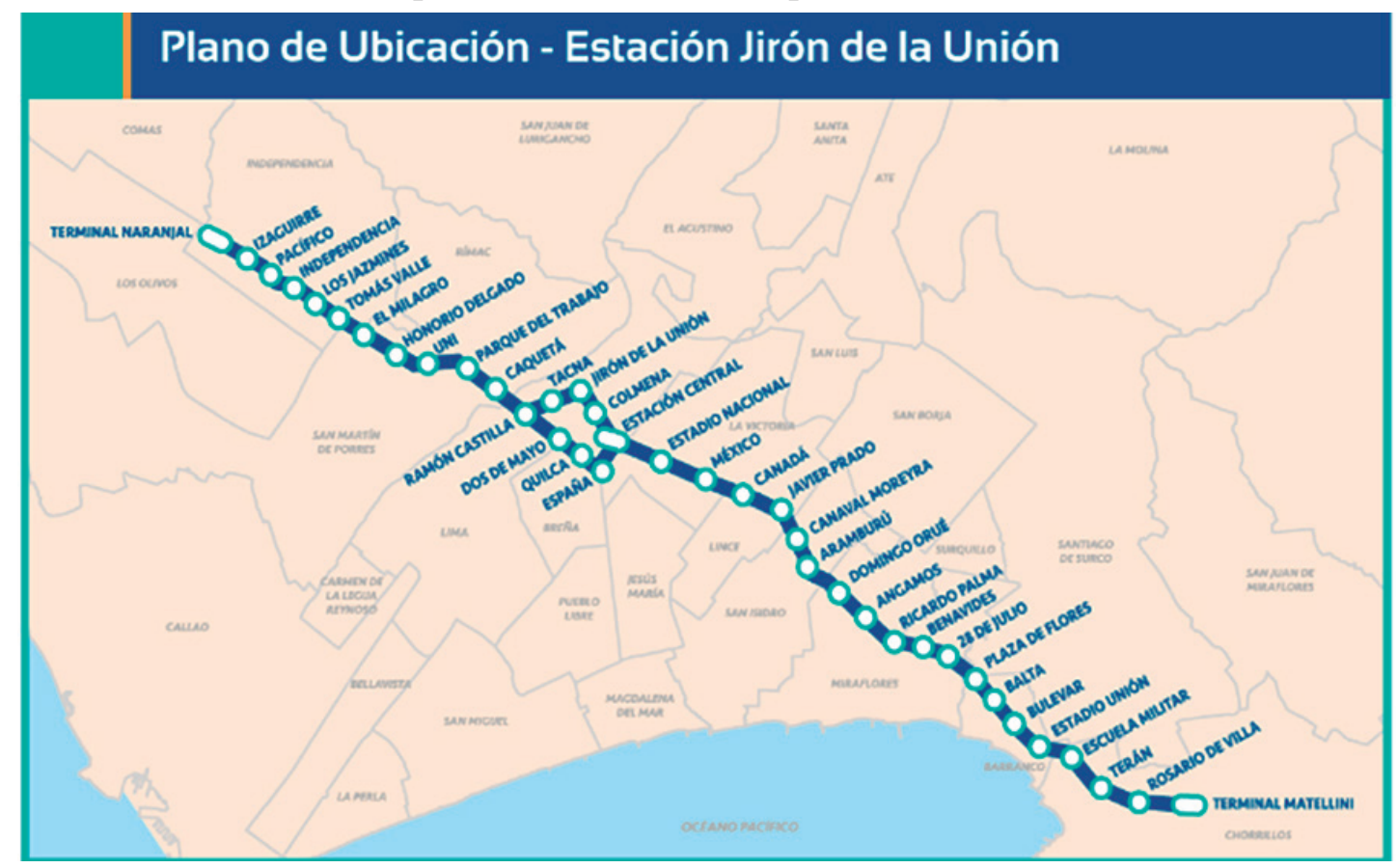

Figura 1. Ilustración de las estaciones del Metropolitano y de los 12 distritos que abarca su ruta Fuente: Página web del Metropolitano

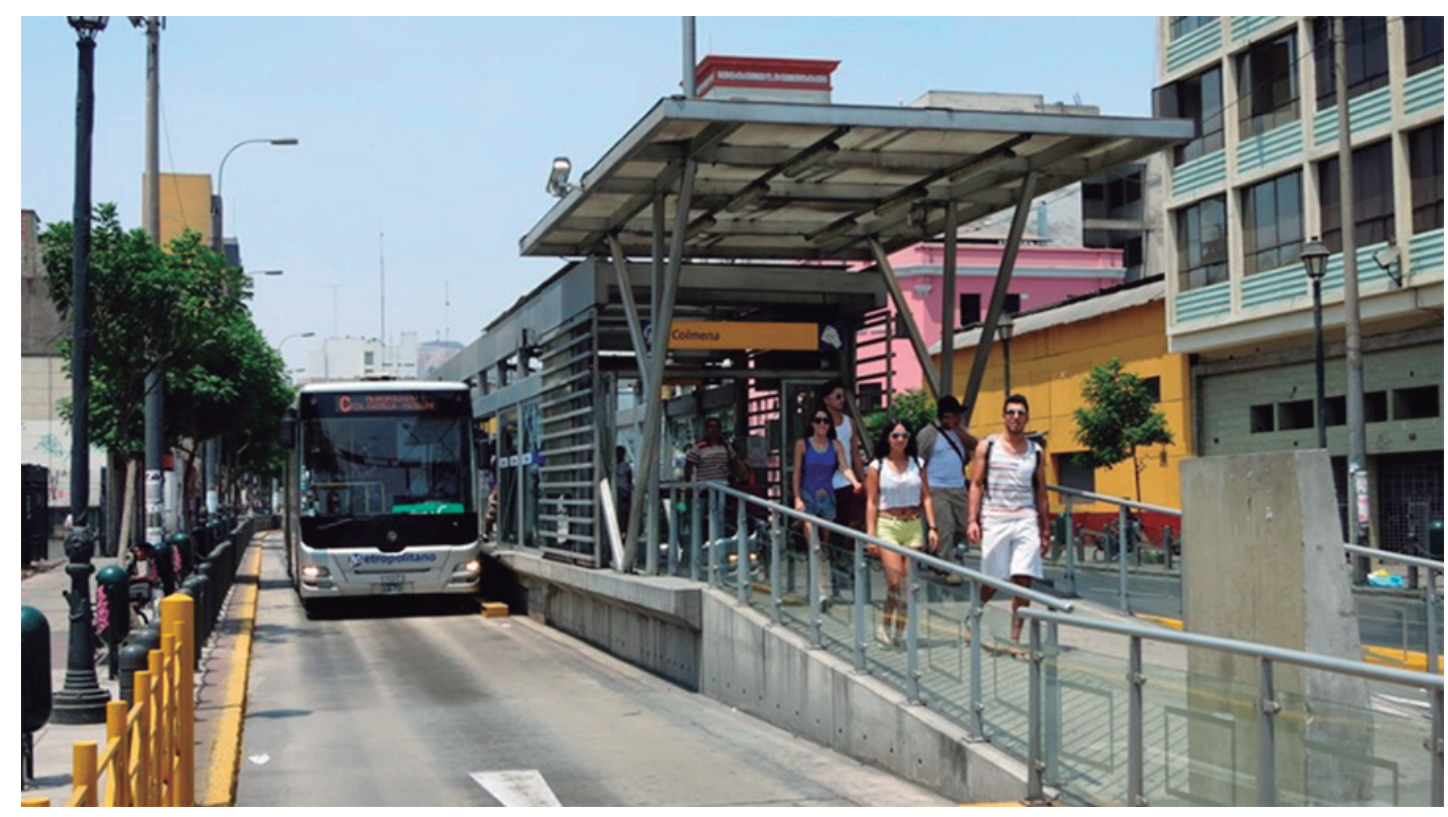

Figura 2. Fotografía de la infraestructura y arquitectura de la estación Colmena (Lima Centro) Fuente: Página web de una guía turística en la ciudad de Lima "Lima City of Kings"

Al no ser pensado este terminal como última estación ${ }^{7}$, se verá congestionada por el recibimiento de usuarios que llegan desde los otros distritos ${ }^{8}$ de Lima Norte; mientras que la otras estaciones solo reciben, por lo general, a usuarios que viven cerca o en el distrito. Creemos que esta diferencia da cabida a que se generen mayores sucesos e interacciones. Sobre esto se puede observar en una reseña ${ }^{9}$ sobre la experiencia y 7 Alcalde de Lima, Jorge Muñoz (2019-presente), sobre el Terminal Naranjal: “La estación de Naranjal, donde se genera la mayor deficiencia, no estaba programada para ser estación final [del Metropolitano], está mal diseñada y lamentablemente por desidia de anteriores administraciones, eso terminó siendo estación final" (Diario El Peruano, vía web: 29/03/2019). 8 Comas, Carabayllo, Ancón y Los Olivos

9 Véase el siguiente enlace que se publicó en la página de Facebook del diario El Comercio: https://elcomercio.pe/somos/ historias/experiencia-metropolitano-esto-es-lo-que-vive-un-ciudadano-que-viaja-de-naranjal-a-miraflores-noticia/ 
significado de transitar y viajar en el Metropolitano por parte de un usuario. Gerardo, el usuario, nos relata su experiencia desde el momento que sale de su casa, cuando llega al Terminal Naranjal (figura 4) -siendo aquí el lugar y momento de suceso e interaccioneshasta abandonar este transporte para dirigirse a su centro de trabajo. De esta manera él nos narra lo que sucedía y de cómo se sentía en todo ese momento de su estadía efímera o de paso, pero simbólica, por este lugar: desde gritos y empujones hasta lograr clasificar tipos de viajeros con tan solo observar las actitudes y comportamientos de los usuarios en el terminal como en el transcurso del viaje en el bus.

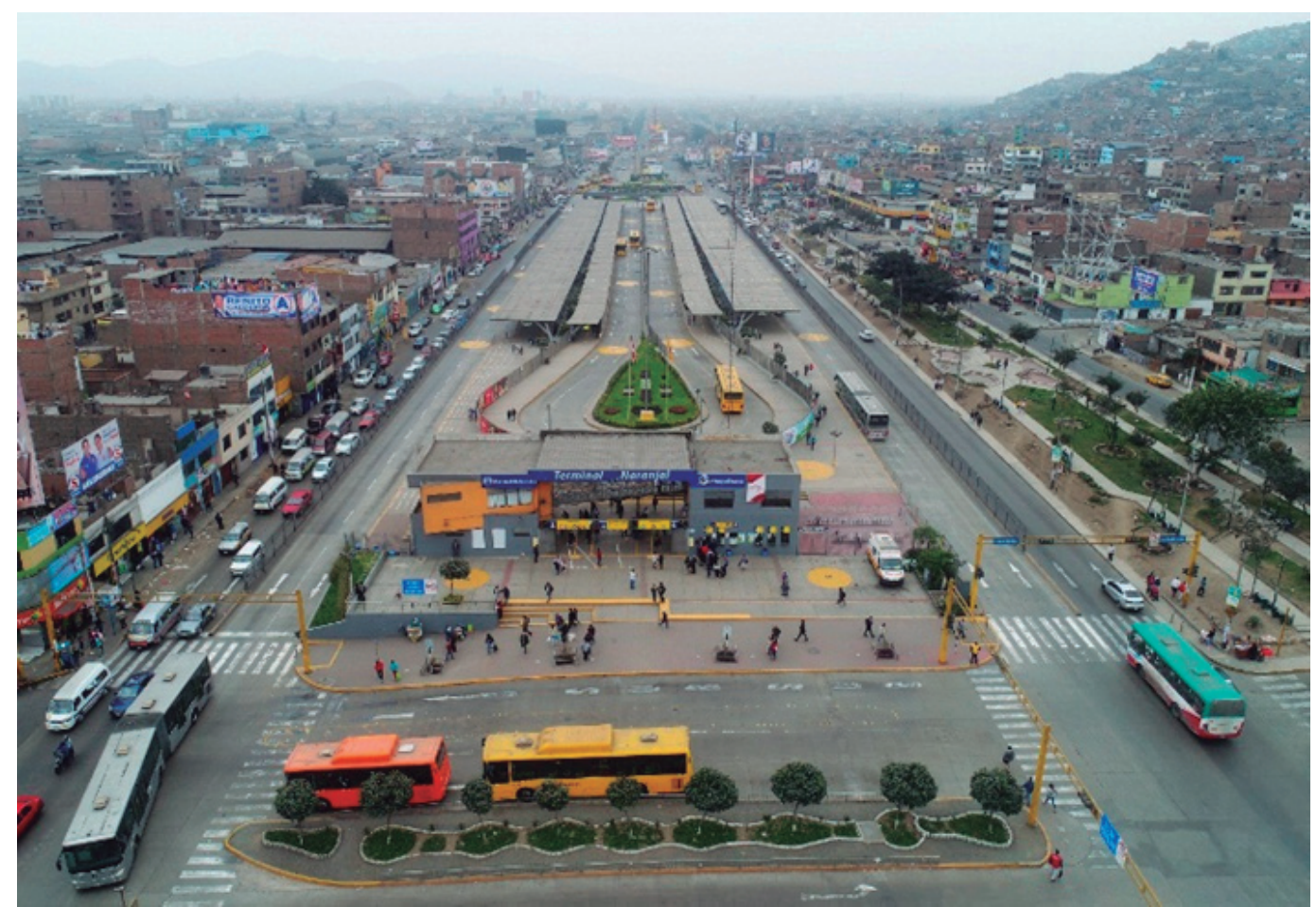

Figura 3. Vista aérea y panorámica del Terminal Naranjal ubicado en el distrito de Independencia (Lima Norte)

Fuente: Página web del Metropolitano

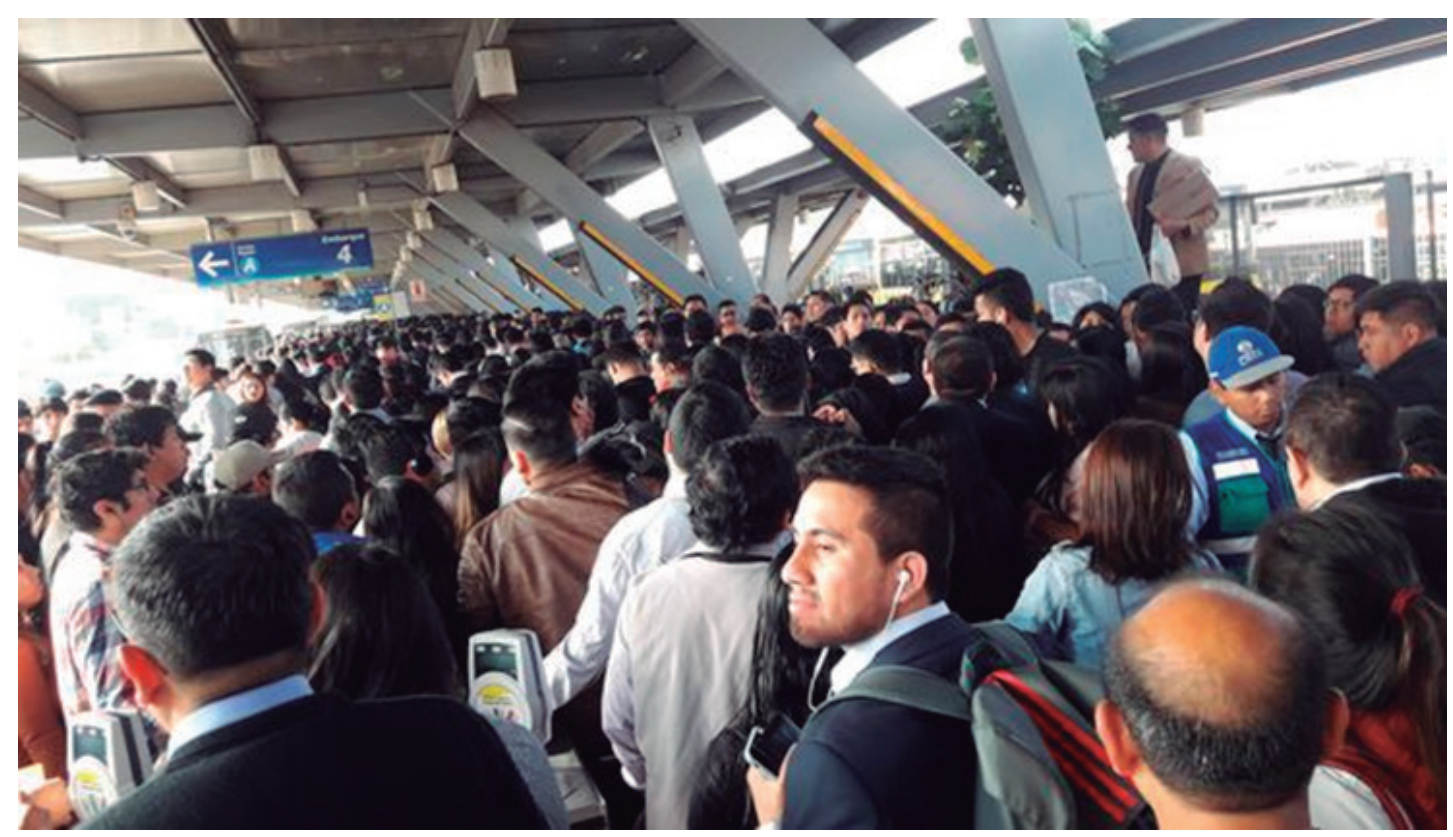

Figura 4. Interior del Terminal Naranjal (hora punta)

Fuente: Página web del diario El Comercio 
Cada detalle de las actitudes que se logran relatar en la reseña es sumamente importante ya que refuerza el significado del Metropolitano como, muy a parte de su función mecánica que es el de transportar, un lugar con una propia dinámica social. En relación con la reseña, creemos que no solo se trata de situaciones efímeras y de tránsito como se piensa comúnmente, sino que también, y reforzando la idea de Gerardo, se trata de un mosaico social y cultural. De tal forma que la estructura social del espacio del Metropolitano puede representarse como una microsituación de la ciudad de Lima.

Ante lo descrito, planteamos la siguiente pregunta como apertura a esta reflexión: ¿Por qué debería ser importante prestar atención a las movilidades e interacciones sociales de los usuarios del Metropolitano, y de qué manera esta pandemia ha repercutido en la interacción de los usuarios y en su relación con el Metropolitano?

\section{Movilidad Simbólica: El Metropolitano como lugar de interacción social}

Antes de analizar la repercusión de la Covid-19 en la dinámica social del Metropolitano, primero iniciaremos con exponer ${ }^{10}$ la situación de la dinámica social hasta antes de la pandemia: Por lo general, por las mañanas del Terminal Naranjal, tanto en el exterior como en el interior del terminal, era un "mar" de usuarios que formaban filas para poder ingresar o para recargar su tarjeta en los puntos de venta automáticos (PVA' s). Luego de haber colocado su tarjeta frente al lector electrónico para así poder ingresar por el torniquete, los usuarios, con preocupación y rapidez, buscaban la fila correspondiente a su ruta de viaje. Esta búsqueda no era sencilla si tenemos en cuenta que en este terminal hay alrededor de 6 o 7 rutas de buses ${ }^{11}$. Una vez que los usuarios encontraban su fila correspondiente, no tendría que pasar más de un minuto para que se vean inmersos en toda una dinámica viva conformada por relaciones intersubjetivas e interacciones sociales que los mismos usuarios producían, aunque esta dinámica sea muchas veces omitida o no reconocida en el momento por los mismos usuarios. Aquella dinámica que exige de una observación participante para ser identificada - o en palabras de Edward T. Hall (2003), de "una cuidadosa observación durante un largo espacio de tiempo y en una gran variedad de situaciones, tomando nota de cada pequeño cambio de información recibido" (pág. 142)-, se conforman por aquellos escenarios o momentos etnográficos que hemos denominado interacciones de "primer tipo" y de "segundo tipo".

Por un lado, los escenarios de primer tipo se caracterizan porque es emitido por el mismo usuario a través de la corporalización de sus emociones y sentimientos, siendo así interactuado y respondido, algunas veces, por otro usuario cercano. En estos escenarios se encuentran: rostros de preocupación e intriga de los usuarios por saber si llegarán a la hora o temprano al centro de trabajo, de estudios o de alguna otra actividad social; gritos y reclamos de los usuarios por la espera de aproximadamente 30 minutos de los buses, o porque algún usuario se ha colado en la fila; insultos, discusiones y golpes leve entre los usuarios por la disputa del sitio al momento de abordar el bus; interacciones de evitamiento entre los usuarios ya que no desean ser tocados o rozados por lo demás; caídas, empujones y golpes al momento al ingresar al bus.

"Todos los días hay reclamos, empujones, gritos, discusiones en las colas y dentro de los buses. La gente hace colas interminables en las que se demora 1 HORA o más para entrar a bus e incluso cuando lo logra va todo aplastado porque no respetan ningún tipo de capacidad máxima en los buses, la gente se mete como pueda y el personal auxiliar no hace nada para controlarlo. Todos los días por culpa del mal servicio que ofrece el Metropolitano

10 Es necesario acotar que la descripción de la dinámica social del Metropolitano esta ambientada en los horarios de mayor agudización social, es decir, en las horas punta.

11 Estas rutas se dividen entre las dos secciones del Terminal Naranjal, es decir, si observamos la figura 4, solo veremos una sección que está ubicado en el margen izquierdo. La otra sección, que se ubica en el margen derecho, se puede observar en la figura 3 que es del mismo terminal. 
le malogra la mañana a todos" (Respuesta extraída de un formulario virtual realizado en Google Forms, 02/07/2020)

Esta respuesta es de un usuario que participo en el formulario virtual ${ }^{12}$ creemos que representa el sentir y el discurso de todas las respuestas recogidas hasta ahora sobre la pregunta acerca de los tipos de escenarios o situaciones que el usuario haya podido identificar en su transitar y viaje por el Metropolitano. Cabe resaltar y recordar que estos escenarios son captados mayormente en horario punta debido a que la carga simbólica es mayor.

Por otro lado, los escenarios de segundo tipo, que a diferencia de solo algunos que hemos mencionado en el primer tipo, son realizados e interactuados por usuarios que comparten algún tipo de vínculo social. De esta manera, la interacción entre ellos es mucho más simbólica por la misma razón que el vínculo mismo lo refuerza.

Estos escenarios han sido de protestas o plantones frente al Terminal Naranjal por parte de diversos colectivos activistas y/o sociales de la población, que son también usuarios del Metropolitano. Planteamos que el vínculo social se encuentra en el "porqué" de sus reclamos y exigencias, como por ejemplo con las protestas y plantones organizado por un grupo de dirigentes de Comas ${ }^{13}$ contra el uso de un Parque Zonal, "Sinchi Roca", que estaba siendo utilizado como "cochera" del Metropolitano y que estaba en planes de agrandarse ${ }^{14}$. Los mismos vecinos-usuarios protestaban también por la oposición de la desaparición de aproximadamente 5000 tipos de especies forestales ${ }^{15}$, de la Av. Universitaria, que sería producida por la ampliación de los tramos y vía exclusiva del Metropolitano hasta Carabayllo. Otros tipos de protestas fueron las de los reclamos por el alza de pasajes ${ }^{16}$, organizado por un grupo conformado por los mismos pasajeros del Metropolitano, y que fueron llevados a cabo tanto en el interior como en el exterior del terminal. De igual manera, esta misma razón de protesta ha sido realizado por estudiantes de secundaria ${ }^{17}$-que usan este servicio de transporte para dirigirse a sus colegios- en diversas ocasiones de este terminal como en la Estación Central. Así mismo, el vínculo puede ser mucho más latente entre los usuarios. Por ejemplo, las estaciones del Metropolitano han sido también escenarios de demostraciones de amor y afectos por las parejas que solían quedarse hablando "pegados" al muro que funcionaba o bien como banca o para arrinconarse. Los besos largos, los abrazos expresivos, las miradas tiernas y enamoradas, los regalos y detalles, los cuerpos juntos al compartir audífonos o ver algún vídeo; las miradas tristes y de melancolía, los rostros alegres o los ojos llorosos son tan solo algunas de las interacciones más significativas y expresivas que podrían notarse en un lugar de paso y de rápidas movilidades como en el Metropolitano

Creemos que estos escenarios mencionados, tanto del primer tipo como del segundo, presentan niveles diferentes de ser percatados como una interacción, por ejemplo, es más fácil que notemos que una pareja se esté besando en los buses o estaciones del Metropolitano u observar un plantón por la reducción de los pasajes; que ver el rostro enojado de un usuario molesto por la demora de los buses. Dichos escenarios agrupados, tantos los del primer y segundo tipo, pueden representar el significado y sentido de movilizarse, interactuar y viajar en este sistema transporte.

12 Como se mencionó, el formulario virtual, aunque haya sido realizado dentro del contexto de la pandemia, sus preguntas no están en base a este contexto; excepto la pregunta 12, en donde se dará a conocer en el siguiente punto.

13 El grupo de dirigente comeños tiene como nombre "Comité de Gestión y Defensas del Parque Zonal Sinchi Roca”. Así mismo, he podido participar en algunas de sus protestas, plantones y volanteos.

14 La extensión sería de 2.2 hectáreas más de las 8 hectáreas que ya venían ocupando. Véase el siguiente enlace: https:// wayka.pe/comas-ampliacion-del-metropolitano-afectaria-parque-sinchi-roca/

15 Para más información de sus actividades y razones de su protesta, véase el siguiente enlace de la página de su Facebook: https://www.facebook.com/defendamoselparquezonalsinchiroca/

16 Véase el siguiente enlace: https://elcomercio.pe/lima/transporte/metropolitano-usuarios-protestan-alza-pasajes-aplica-hoy-noticia-575156-noticia/

17 Véase el siguiente enlace: https://larepublica.pe/sociedad/2019/11/28/metropolitano-de-lima-reportan-disturbios-en-estacion-central-por-protesta-escolar-video/ 
Por una parte, esto se puede entender como el imaginario colectivo o urbano (Lindón, 2012 y Hiernaux, 2012) que se ha proyectado y generado en el espacio del Metropolitano, es decir, como un lugar de estrés, molestias, disputas, protestas, reclamos, etc. Es como aquellos lugares de miedo que Alicia Lindón (2009) analiza para la relación cuerpo-lugar, donde el "miedo no solo da sentido al lugar sino también se corporiza" (p. 10), así como también al tratar con los lugares de violencia. Esto quizás puede ayudarnos a entender del porqué de esas emociones y sentimientos que se han podido identificar mediante la observación de los diversos cuerpos y comportamientos entre los usuarios del Metropolitano.

Por otra parte, las interacciones de segundo tipo, es decir de las que tenían algún vínculo social, no solo tuvieron como escenario las estaciones del Metropolitano. Por el contrario, algunas de estas protestas (escolares, vecinales y de colectivos) fueron también presenciadas e incluidas en diferentes marchas y protestas sociales en la ciudad de Lima. Este hecho nos puede dar a entender que lo que ocurre en el Metropolitano no está desligado de los otros problemas sociales y urbanos que se experimenta en la dinámica cotidiana de la ciudad. En este sentido, se comprende al Metropolitano como una institución o lugar que acontece diversos escenarios de interacciones, que esta interconectada con otras instituciones y lugares con sus propias interacciones, que integrados permiten el funcionamiento de la dinámica urbana de la ciudad. O en palabras de Alicia Lindón (2009):

En esos haceres encadenados de múltiples sujetos que convergen por instantes en cierto lugares y ciertos fragmentos de tiempo, y luego se distancian y protagonizan nuevas convergencias espacio-temporales con otros sujetos y en otro lugares, se va desarrollando la construcción socio-espacial de la ciudad, de manera permanente, fragmentada y al mismo tiempo, interconectada. Es una construcción fragmentada porque en cada lugar y en cada instante ocurren fenómenos singulares, con vida propia, que le van marcando rumbos precisos a la ciudad. (p. 13)

Con esto pretendemos dar a entender también que la interacción no se debe entender únicamente entre persona y persona, sino que también entre la persona y el lugar como fue con el caso de las interacciones de segundo tipo. Es decir, los usuarios se encuentran en una interacción y relación contractual con el servicio del Metropolitano ya que consideran que el servicio es como "no debería ser" y no es como "debería ser" (ordenado, tranquilo, con más espacio, con una tarifa justa, menos estrés, etc.). En otras palabras, la interacción contractual hasta contradictoria es el resultado entre el tipo de servicio que provee el Metropolitano y la experiencia que viven los usuarios rutinariamente por este servicio. Aunque esta interacción sea efímera, de paso y transitoria, no deja de ser sumamente relevante porque nos hacer entender al Metropolitano en un nivel "microcultural" (Hall, 2003), para así poder pensar, en un nivel más macro, en el tipo de ciudad en que nos movilizamos e interactuamos diariamente.

Sumado a esto, la interacción contractual y contradictoria entre los usuarios y el lugar del Metropolitano, también nos permite observar al Metropolitano como un espacio político e ideológico en torno al cómo debería ser la administración y funcionamiento de este transporte. Mas aún si tenemos en cuenta que el Metropolitano es consciente de las condiciones que dispone en la experiencia del viaje. Por algo, en el 2019, no intentaron apaciguar la demora y espera de los buses colocando música clásica ${ }^{18}$ en el Terminal Naranjal, como si esto fuese un tranquilizador en horarios punta donde lo único que se desean los usuarios es llegar lo más antes al lugar donde se desarrollan socialmente. Siendo así que esta iniciativa del Metropolitano no tuvo eficacia y no duro mucho.

18 Véase el siguiente enlace: https://elcomercio.pe/lima/sucesos/metropolitano-difunden-musica-clasica-estacion-naranjal-relajar-pasajeros-noticia-nndc-643805-noticia/ 
En conclusión, tener en cuenta estas interacciones de primer tipo y de segundo tipo es de gran relevancia si pensamos que pueden ser tomadas en cuenta para las futuras planificaciones urbanas o para las políticas de transporte, y así poder reconfigurar el significado de movilizarnos e interactuar en la ciudad de Lima desde las prácticas cotidianas. En otras palabras, "repensar la forma de intervenir desde límites administrativos, a definir formas de intervención a partir de cómo las personas habitan su territorio de manera cotidiana" (Jirón, 2017, p.278). De igual forma, tanto los primeros y segundos tipos de interacciones, en general, conforman la dinámica interna que aflora y se produce en el Metropolitano como lugar antropológico. En este sentido, no estamos tan conforme con el concepto "No-Lugares" (1998) ${ }^{19}$ del antropólogo francés Marc Augé al considerar como lugar no antropológico (Augé, 1998) a estos lugares de paso y de tránsito, más aún si se tiene en cuenta todo lo descrito y analizado hasta el momento. Contrario a esto, todo ese significado antropológico del Metropolitano integra a ese "verdadero espíritu de la ciudad" o "personalidad de la ciudad", que Daniel Hiernaux (2012, p.90) hace referencia al abordar sobre los imaginarios y las dimensiones subjetivas de la ciudad.

\section{Movilidades e interacciones en el Metropolitano en tiempos de pandemia}

Antes de comenzar este punto es necesario contextualizar brevemente el presente escenario de pandemia: La actual pandemia por la Covid-19 ha generado que los países del mundo hayan planteado diversas medidas para combatir este virus. Sin embargo, esto ha repercutido en sus diversas actividades sociales de cada país. Nuestro país, Perú, no ha sido ajeno a este contexto y desde la quincena de marzo se decretó en estado de emergencia ${ }^{20}$, generando medidas como el aislamiento y la inmovilización social nocturna. Esto produjo que algunas actividades sociales se hayan visto detenidas en su funcionamiento cotidiano, lo que conllevo a que estas actividades sean llevadas a realizarse en el plano virtual desde el hogar. Por otro lado, aquellos lugares de puente y de paso donde cientos de personas transitaban en el transcurso del día para dirigirse a sus actividades se han visto reducido en número, tal y como se observó en las estaciones del Metropolitano.

Ahora bien, todos estos escenarios mencionados en el punto anterior fueron registrados y observados hasta antes de la pandemia ${ }^{21}$, de tal manera, como se ha dicho en líneas arriba, que el contexto actual haya repercutido en la experiencia de movilizarse e interactuar en el Metropolitano. Creemos que es así por una razón principal: las nuevas reglas y medidas de viajar "del" Metropolitano - "del" porque es entendida como una institución que conforma y administra parte del espacio urbano de la ciudad de Lima- por la pandemia. Estas nuevas reglas comenzaron a ser practicadas y tomadas en serio desde que uno de los requisitos para usar el Metropolitano fuera el uso obligatorio de la mascarilla, así como luego fue con los protectores faciales ${ }^{22}$. Por otro lado, el Metropolitano ha implementado nuevas señalizaciones para los usuarios como la separación de un metro de distancia para abordar el bus y en el uso de los asientos que no tengan la " $\mathrm{X}$ " en los buses, la continua desinfección de los buses y de las estaciones, así como de la provisión de desinfectantes con alcohol en los servicios higiénicos

19 Esta disconformidad se explica porque no consideramos que la única relación (y contractual) que se experimenta entre los pasajeros o "clientes de paso" (Augé 1998: 108) -si entendemos por clientes a la acción de comprar un acceso para poder ingresar a una estación de transporte- y el no lugar sea el ticket, el pasaje o la tarjeta del Metropolitano para el caso nuestro. Además, que no compartimos que sean las tres características (no identidad, no relacional, no histórico) de un "no-lugar" como determinantes para que un lugar sea un "no-lugar".

20 Véase el siguiente titular: https://gestion.pe/peru/politica/coronavirus-en-peru-gobierno-anuncia-cuarentena-obligatorio-por-15-dias-por-coronavirus-noticia/ .

21 La observación ha sido continua desde finales del 2018 cuando me comenzaba a preguntar sobre mi experiencia al viajar en el Metropolitano. No obstante, no fue suficiente hasta que comencé a descubrir sobre el estudio de las interacciones sociales que el interés aumentó.

22 Véase el siguiente enlace del comunicado de la Municipalidad de Lima: http://www.munlima.gob.pe/noticias/ item/40362-comunicado-desde-manana-uso-del-protector-facial-sera-obligatorio-en-metropolitano-y-corredores-complementarios 
Siendo esto solo algunas de las nuevas medidas ${ }^{23}$ que se han ido agregando y modificando en la experiencia y forma de viajar en el Metropolitano. Tanto los nuevos implementos como el uso obligatorio para viajar y transitar para los usuarios, así como las nuevas medidas y normas establecidas por el Metropolitano (al administra parte del espacio urbano de la ciudad puede en su facultad implantar nuevas reglas y normas de transitar); son los medios por donde la pandemia del Covid-19 ha repercutido en esta microsituación de la ciudad.

Para poder enriquecer el análisis sobre la repercusión de este contexto, consideramos tener en cuenta los conceptos "sujeto cuerpo" y "sujeto sentimiento", nuevamente de la geógrafa humana Alicia Lindón, para pensar sobre las interacciones en este contexto. Para la autora, los cuerpos de los sujetos o personas están en estrecha relación con los lugares que transita y que forman parte. En dicha relación los cuerpos son un medio para expresar y emitir las emociones intersubjetivas que los sujetos experimentan y sienten mientras se movilizan e interactúan con otros en un lugar en particular. Para ello, la autora cita algunos ejemplos de los lugares de miedo, de violencia, de denuncias, de memoria; así como también de los lugares de tránsito y de paso ${ }^{24}$. En palabras de Alicia Lindón:

La relación de una práctica en cierto lugar puede movilizar en el sujeto, afectos por diversas cuestiones. Por ejemplo, por recuerdos agradables del lugar, por recuerdo de situaciones gratas vividas en el lugar, por temores que relaciona directamente con el lugar, por miedos, por recuerdos dolorosos vinculados al lugar, por sentimientos de inseguridad emanados del estar en ese lugar... Estas componente afectivas y emocionales no sólo vienen articuladas en una práctica espacializada, también suelen ocurrir que esos estados emocionales impulsan al sujeto a realizar otras prácticas (Lindón 2009, p.12).

Con relación a la cita anterior, pensamos que los sentimientos y emociones corporalizados en los sujetos hasta antes de la pandemia se han podido agudizar debido a ese miedo mayor sobre el contagio de la Covid-19. Por otra parte, el actual contexto también ha representado, para un sector de usuarios, una oportunidad para reflexionar y pensar sobre las condiciones de transporte que el Metropolitano ha venido ofreciendo hasta el momento.

“Creo que esta pandemia podría significar una oportunidad para aplicar mejor los programas de organización de las estaciones. Hoy existe el miedo de acercarse al otro, pero impera la necesidad de llegar a tiempo al trabajo. Creo que un trabajo en conjunto puede ayudar a superar esta crisis, tienen que involucrar más a los ciudadanos en la organización de las estaciones" (Formulario virtual realizado por Google Forms, 2 de julio del 2020).

"Creo que se la ha devuelto prestigio al servicio. Antes no había respeto al cliente cuando éste abordaba el bus pues prácticamente el servicio parecía abarrotado como las combis. Creo que un servicio controlado es el que siempre se debió ofrecer, no creo que haya cambiado, lo que creo es que ahora es como siempre debió ser, fuera de la cantidad limitada de pasajeros que abordan cada bus" (Respuesta extraída de un formulario virtual realizado en Google Forms, 06/07/2020).

Si bien es cierto que se ha visto reducida el número de usuarios que viajan en el Metropolitano, no parece que las prácticas e interacciones hasta antes de la pandemia se hayan omitido del todo; por el contrario, tal y como lo menciona un usuario:

"Creo que la situación que se vive en las estaciones es la misma con o sin mascarillas, puesto que la gente sigue haciendo las mismas colas largar esperando buses cuando se debería aumentar los buses para que la gente no esté apretada" (Respuesta extraída de un formulario virtual realizado en Google Forms, 06/07/2020).

23 Véase el siguiente enlace del comunicado de la Municipalidad de Lima: http://www.munlima.gob.pe/noticias/ item/39692-municipalidad-de-lima-adopta-medidas-de-prevencion-en-el-metropolitano

24 Lo cual lleva a la autora a contradecir el concepto "no-lugares" de Marc Augé (Lindón, 2009: 11). 
Por otro lado, se considera importante tener en cuenta los nuevos imaginarios sociales producto de esta pandemia, que postulamos que ha influido en la intersubjetividad de los usuarios del Metropolitano. Estos nuevos imaginarios sociales están conformados por esa diversidad de explicaciones, noticias, informes, estudios y enunciados oficiales sobre el Covid-19. Incluso también los comentarios virtuales, las fakenews, los consejos de recetas caseras "anti-covid", los vídeos explicativos para protegerse de esta pandemia, etc. Es decir, esas cadenas de informaciones (oficiales y no oficiales) que se comparten por medio de las redes sociales y los medios de comunicación, y que tienen un gran alcance en las ideas, sentimientos, pensamientos; en general, en lo subjetivo de la persona-usuario.

Pero ¿Cómo puede entenderse un imaginario social ${ }^{25}$, que es muy general y que no solo abarca en un espacio delimitado ${ }^{26}$ como el Metropolitano? Pues bien, Daniel Hiernaux (2012) explica esto desde la condición geográfica empleando el ejemplo del miedo. Para ello, el autor coloca al miedo en dos escenarios distintos, el primero como producto de un cataclismo natural y que puede repercutir en toda una nación entera (como en Japón) o por el terrorismo y la inseguridad generalizada (Hiernaux, 2012: 92) de un país. Hasta este punto, por estar el miedo en un nivel macro, el autor lo entiende como un imaginario social (general) debido a que es compartido por toda la población. Por otro lado, en el segundo escenario, el autor entiende al miedo como producto de los "comportamientos urbanos" (Hiernaux 2012, p.92), como, por ejemplo, la segregación social de un espacio geográfico en particular que puede representar y simbolizar el miedo. Siendo este factor geográfico y los comportamientos urbanos que se desenvuelven en un espacio en particular que el autor los considera como imaginarios urbanos.

Si bien, nuestro caso no es categorizado como una segregación social, no obstante, sería propicia emplear esta lógica geográfica para analizar la reconfiguración de los imaginarios sociales en las estaciones de este sistema de transporte como resultado de la pandemia. En otras palabras, como una prolongación o proyección representada en los imaginarios urbanos en el espacio geográfico-vial del Metropolitano que ocupa parte de la ciudad de Lima. En ese sentido, postulamos que toda esa carga receptiva (los imaginarios) que han obtenido por medio de esas cadenas de informaciones relacionadas a la pandemia serán puesta en escena desde que ingresan por el torniquete al Terminal Naranjal (recordemos que se ha empleado a esta estación como ejemplo que incluye a todas las otras estaciones).

Aunque en realidad desde que se abandona el hogar, la calle misma será de otra experiencia. Si antes de la pandemia, el objetivo luego de salir de casa era llegar al lugar donde desenvolvemos nuestras actividades que consideramos importantes socialmente, de tal manera que en todo ese tránsito el estado anonimato ${ }^{27}$ sería lo más característico. Ahora con esta pandemia, creemos que el objetivo no es solo continuar con las actividades, es decir, llegar los lugares donde uno se desenvuelve y desarrolla; sino que, además, se tendrá que prestar atención, de cierta manera, a la otra persona que se encuentra próxima al espacio que la persona ocupa. Creemos que es por la razón que pueda ser contagiado mediante otra persona o porque pueda rozar alguna cosa u objeto que ya ha sido tocado anteriormente, y que signifique una

25 Entendemos el imaginario social como lo define Alicia Lindón (2012): “[...] los imaginarios constituyen un patrimonio de ideas y de imágenes mentales acumuladas, recreadas y tejidas en una trama, por parte del individuo en el curso de su socialización, es decir, a lo largo de toda su vida. Ningún individuo elabora estas construcciones de sentido aislado de los otros, sino en diálogo y en interacción con los otros, es decir, intersubjetivamente, y valiéndose de herramientas socialmente construidas, como el lenguaje" (pág. 66).

26 En el sentido que ocupa parte del espacio geográfico-vial de la ciudad de Lima.

27 En el sentido que no se conoce quién es la otra persona, sujeto social, pasajero, transeúnte o usuario que se encuentra en la calle o en el lugar por donde se transita y se dirige uno. Por otro lado, considero a los aportes de Erving Goffman $(1970,2001)$, Manuel Delgado $(1999,2011)$ y Marc Augé $(1998,2005)$ como cercanos en explicar este estado de anonimato que las personas (o las caras como diría Goffman) adquieren en los lugares o escenarios de rápida movilidad y de paso de la ciudad. 
posibilidad de contagiarse ${ }^{28}$. Es esta atención sobre el otro que como resultado reduce el valor simbólico del estado de anonimato que la persona se solía "proteger" y pasar desapercibido mientras transitaba por las calles y los lugares de paso o puente.

Dentro de este marco, en nuestro caso, los usuarios al prolongar toda esa carga receptiva en el terminal reconfigurarán la forma de transitar porque se tendrá en cuenta la posibilidad de ser contagiado en cualquier momento o situación en la interioridad del terminal. Esta reconfiguración del sentido de transitar y viajar por este transporte no solo se refuerza por las normas y medidas que el Metropolitano ha determinado para los usuarios: la separación de un metro en la fila de abordar, el uso obligatorio a los usuarios para con sus implementos (mascarilla y protector fácil). Sino que también depende de la función de quienes trabajan orientando a los pasajeros o usuarios de este transporte: los orientadores. Para ello, finalmente, proponemos entender, simbólicamente, a los orientadores como medios o puentes por donde el Metropolitano reafirma sus nuevas medidas y normas implementadas sobre los usuarios, y así reafirmar también su autoridad como institución. Esto se da en la medida que los orientadores presten mayor atención y vigilancia hacia los usuarios, para que de esta manera se verifique lo dictado por la institución del Metropolitano.

\section{A modo de conclusión}

Las movilidades e interacciones de los usuarios por este servicio de transporte masivo, urbano y moderno de la ciudad de Lima son sumamente importante a la hora de pensar en la ciudad y en la manera en cómo sus habitantes se integran y se movilizan cotidianamente. Desde este lugar y escenario, el Metropolitano, se ha buscado, en primer lugar, reflexionar y aportar al análisis de lo que sucede en la interioridad de este sistema de transporte hasta antes de la pandemia. Siendo las interacciones de primer tipo y de segundo tipo las pruebas empíricas que el Metropolitano simboliza y significa, muy a parte del fin mecánico que es el de transportarse de un punto a otro o de una estación a otra, un lugar que desenvuelve una propia dinámica interna originado por las interacciones entre los usuarios. Dicho valor simbólico de este sistema de transporte no sería posible si no entendemos y observamos a los cuerpos de los usuarios, como puentes donde las emociones y sentimientos se corporalizan en todo el momento que signifique estar en este lugar de transporte. De tal manera que la cotidianidad de estas corporalidades origina, justamente, ese sentido simbólico y significativo del Metropolitano.

En segundo lugar, dichas interacciones que se venían practicando desde antes de la pandemia, han sido analizadas dentro del contexto actual de la Covid-19. Observándose así las repercusiones en las movilidades e interacciones de los usuarios debido a los nuevos imaginarios urbanos e intersubjetivos que han calado en el sentir y actuar como resultado del contexto global. Sumado a ello, las nuevas normas y medidas impuestas por el Metropolitano, que es reforzada y reafirmada en la vigilancia y observación de los orientadores hacia los usuarios

Por último, es necesario acotar que tanto antes de la pandemia como en el presente contexto, las movilidades e interacciones que se desenvuelven en este transporte urbano, son importante tenerlos en cuenta en los proyectos o políticas urbanas para así generar mejores condiciones de movilizarse, interactuar y transitar por la ciudad. Más aún en este tipo de sociedad donde la experiencia cotidiana de las personas no es tomada en cuenta dentro de los marcos de los profesionales de la ciudad (arquitectos, urbanistas, ingenieros, etc.), ya sea por diversas razones políticas, económicas o por simple desconocimiento y falta crítica en pensar en el tipo de ciudad en que queremos vivir.

28 Esto se explica del porque muchas personas cuando viajan en el Metropolitano portan en sus mochilas, carteras, etc., un frasco pequeño que contiene alcohol. O que usen, muy a parte de los implementos obligatorios, encima de sus vestimentas un traje de plástico para sentirse más seguros y protegidos en esta pandemia. 


\section{Referencias Bibliográficas}

Aguilar, Miguel A. (2013). Ciudad de interacciones: el cuerpo y sus narrativas en el metro de la ciudad de México. En Miguel A. Aguilar y Paula Soto (coord). Cuerpos, espacios y emociones. Aproximaciones desde las ciencias sociales. (1-23). México: Porrua, Universidad Autónoma Metropolitana-Iztapalapa. http://www.academia.edu/15631132/ Ciudad_de_interacciones_el_cuerpo_y_sus_narrativas_en_el_metro_de_la_ciudad_ de_M\%C3\%A9xico?auto=download

Augé, Marc. (1998). Los <no lugares>. Espacios del anonimato. Una antropología de la sobremodernidad. Barcelona: Gedisa.

Augé, Marc. (2009). El viajero subterráneo. Un etnólogo en el metro. Xalapa, México: AL FIN LIEBRE ediciones digitales. http://alfinliebre.blogspot.com/

Becker, Howard. (2009). Trucos del Oficio: Cómo conducir su investigación en ciencias sociales. Buenos Aires: Siglo XXI.

Becker, Howard. (2014). Outsiders: Hacía una sociología de la desviación. Buenos Aires: Siglo XXI.

Becker, Howard. (2016). Cómo fumar marihuana y tener un buen viaje: Una mirada sociológica. Buenos Aires: Siglo XXI.

Delgado, Manuel. (1999). El animal público. Hacia una antropología de los espacios urbanos. Barcelona: Anagrama.

Delgado, Manuel. (2011). El espacio público como ideología. Madrid: Catarata

Grimaldo, Christian Omar. (2020). Hacia un paradigma transeúnte: el abordaje de la cultura a partir de los trayectos cotidianos. Encartes, Vol 3 ( $\left.\mathrm{N}^{\circ} 5\right)$, 1-9. https://encartesantropologicos. $\mathrm{mx} /$ grimaldo-transeunte-cultura-trayectos/

Foucault, Michael. (2010). El cuerpo utópico. Las Heterotopías. Buenos Aires: Nueva Visión.

Goffman, Erving. (1970). Ritual de la interacción. Buenos Aires: Tiempo Contemporáneo.

Goffman, Erving. (2001). La presentación de la persona en la vida cotidiana. Buenos Aires: Amorrortu.

Hall. Edward T. (2003). La dimensión oculta. Mexico: Siglo. XXI.

Hernández, José. (2017). El metro de la ciudad de México: heterotopías y prácticas homoeróticas. En Galia Cozzi y Pilar Velázquez (coord), Desigualdad de género y configuraciones espaciales (274-290). México: Universidad Nacional Autónoma de México; Centro de Investigaciones y Estudios de Género, Programa Universitario de Estudios sobre la Ciudad; Instituto de Geografía.

Hiernaux, Daniel. (2012). Los imaginarios urbanos: Una aproximación desde la geografía urbana y los estilos de vida. En Alicia Lindón y Daniel Hiernaux (dirs.), Geografías de lo imaginario (87-116). Barcelona: Anthropos Editorial, México: Universidad Autónoma Metropolitana - Iztapalapa. Div. Ciencias Sociales y Humanidades.

Hiernaux, Daniel. y Alicia Lindón. (2012). Geografías de los imaginarios. Barcelona: Anthropos Editorial; México: Universidad Autónoma Metropolitana - Iztapalapa. Div. Ciencias Sociales y Humanidades.

Ingold, Tim. (2017). ¡Suficiente con la Etnografía!. Revista Colombiana de Antropología. Vol 53 (ํㅜ 2), 143-159. https://www.redalyc.org/pdf/1050/105052402007.pdf

Jirón, Paola. (2017). El hábitat residencial observado desde la movilidad cotidiana urbana. En Imilan, W; Larenas, J; Carrasco, G y Riversa, S (eds.). ¿Hacia dónde va la vivienda en Chile?: nuevos desafíos en el hábitat residencial (269-279). Santiago de Chile: INVI, MINVU, Adrede. http://repositorio.uchile.cl/handle/2250/144894

Lefebrve, Henri. (2013). La producción del espacio. Madrid: Capitán Swing.

Lindón, Alicia. (2009). La construcción socioespacial de la ciudad: el sujeto cuerpo y el sujeto sentimiento. Revista Latinoamericana de Estudio sobre Cuerpos, Emociones y Sociedad, Vol 1 ( $\mathrm{N}^{\circ}$ 1): 06-20. https://www.redalyc.org/articulo.oa?id=273220612009 
Lindón, Alicia. (2012). ¿Geografías de lo imaginario o la dimensión imaginaria de las geografías del Lebenswelt?. En Alicia Lindón, Daniel Hiernaux (dirs.). Geografías de lo imaginario (65-86). Barcelona: Anthropos Editorial, México: Universidad Autónoma Metropolitana - Iztapalapa. Div. Ciencias Sociales y Humanidades

Nava, Alfredo (2019). Interacción, construcción del espacio social y sexualidad en el Metro de la Ciudad de México. Revisa Brasileira de Estudios Urbanos e Regionais, Vol 21 (№3) 524-545. https://www.scielo.br/scielo.php?pid=S2317-15292019000300524\&script=sci_arttext

Restrepo, Delgado. (2018). Etnografía. Alcances, técnicas y éticas. Lima: Fondo Editorial de la Universidad Nacional Mayor de San Marcos.

Toledo, Diana. (2014). Los viajes invisibles: El transporte público en la madrugada limeña. La Colmena ( $\mathrm{N}^{\circ}$ 7). 73-85. http://revistas.pucp.edu.pe/index.php/lacolmena/article/ view/12514

Vivando, Rafael. (2019). Línea 1 del Metro de Lima como espacio social para la construcción de una ciudadanía globalizada. Ponto Urbe, Vol 24. 1-16. https://doi.org/10.4000/ pontourbe.6675 